\title{
Evaluation of the risk of Iymphomagenesis in xenografts by the PCR-based detection of EBV BamHI $W$ region in patient cancer specimens
}

\author{
Junko Mukohyama ${ }^{1,2}$, Dai Iwakiri ${ }^{3}$, Yoh Zen ${ }^{4}$, Toru Mukohara ${ }^{5,6}$, Hironobu Minami ${ }^{5,6}$, \\ Yoshihiro Kakeji ${ }^{2}$, Yohei Shimono ${ }^{1,5}$ \\ ${ }^{1}$ Division of Molecular and Cellular Biology, Kobe University Graduate School of Medicine, Kobe, Japan \\ ${ }^{2}$ Division of Gastrointestinal Surgery, Kobe University Graduate School of Medicine, Kobe, Japan \\ ${ }^{3}$ Division of Clinical Virology, Kobe University Graduate School of Medicine, Kobe, Japan \\ ${ }^{4}$ Department of Diagnostic Pathology, Kobe University Graduate School of Medicine, Kobe, Japan \\ ${ }^{5}$ Division of Medical Oncology/Hematology, Kobe University Graduate School of Medicine, Kobe, Japan \\ ${ }^{6}$ Cancer Center, Kobe University Hospital, Kobe, Japan \\ Correspondence to: Yohei Shimono, email: yshimono@med.kobe-u.ac.jp \\ Keywords: patient-derived tumor xenograft, lymphomagenesis, Epstein-Barr virus, colorectal cancer, BamHI W region \\ Received: April 26, 2016 \\ Accepted: June 13, 2016 \\ Published: June 29, 2016
}

\section{ABSTRACT}

Establishment of patient-derived tumor xenografts (PDXs) is hampered by lymphomagenesis mostly caused by the latently-infected Epstein-Barr virus (EBV) contained in patient cancer tissues. However, the character of patient tissues that result in lymphomagenesis after xenotransplantation is not elucidated. In this study, we analyzed the patient colorectal cancer (CRC) tissues and the PDXs established by their xenotransplantation. We found that 2 of 9 (22\%) PDX tumors were EBVassociated human diffuse large $B$ cell lymphoma which was formed by clonal proliferation of human B-cell lymphocytes, were strongly positive for EBER-ISH, and were classified as type III latency. Expression of EBV genes and RNAs, such as EBNAs, LMP1, EBER and EBV-associated microRNAs in patient CRC tissues were unlikely to be associated with lymphomagenesis in PDXs. In contrast, the positive PCR-based amplification of BamHI W region, a major internal repeat in EBV genome, in the patient CRC tissues was correlated with lymphomagenesis in PDXs. These results suggest that the detection of the EBV BamHI $W$ region in the patient surgical specimens will be an effective way to predict the risk of lymphomagenesis in PDXs before xenotransplantation.

\section{INTRODUCTION}

Patient-derived tumor xenografts (PDXs) are established by xenotransplantation of surgically resected human cancer specimens into immunodeficient mice, such as a nonobese diabetic/severe combined immunodeficientcy (NOD/SCID) mouse which is deficient in both innate and adaptive immunities, and an NOD/ SCID/IL-2rgnull (NSG) mouse which further lacks NK cell activity. PDX tumors are able to mirror patients' histopathological and genetic profiles, can metastasize to distant organs, and are characterized by the presence of a cancer stem cell (CSC) population [1-3]. Furthermore, PDXs are an attractive preclinical model to investigate therapeutic response, cancer resistance, metastasis and biomarkers $[4,5]$.

It is reported that the engraftment of human cancer specimens into an immunodeficient mouse unexpectedly resulted in the formation of lymphoma instead of their corresponding cancers, such as lung, liver, gastric, bladder, breast and prostate cancers [6-10]. The incidence of lymphomagenesis among the established PDXs differ widely depending on the cancer types; for example, 1/30 PDXs (3.3 \%) in colorectal cancer (CRC), 11/21 (56\%) PDXs derived from 16 patients in liver cancer, and 8/10 PDXs $(80 \%)$ in prostate cancer $[7,8,10]$. In addition, transplantation of the same cancer specimen resulted in the formation of a lymphoma PDX in one mouse and a 
cancer PDX in another mouse [9]. Pathological analyses of 26 lymphoma PDXs revealed that 23 PDXs were EpsteinBarr virus (EBV)-associated human B cell lymphoma, and the remaining 3 PDXs were lymphoma of mouse origin [8].

EBV is a member of human-restricted herpes virus. While EBV is well known as the causative agent of infectious mononucleosis, it is associated with various malignancies, such as Burkitt's lymphoma, Hodgkin's lymphoma, nasopharyngeal cancer, gastric cancer, and post-transplant lymphoproliferative disorders (PTLDs) [11]. PTLDs express $12 \mathrm{EBV}$ latent gene products, including 6 EBV nuclear antigens (EBNA1, 2, 3A-C and leader protein), 3 latent membrane proteins (LMP1, 2A and 2B), BamHI-A rightward transcripts (BARTs) and 2 EBV-encoded small RNAs (EBER1 and 2) [11]. EBV efficiently infects resting B cells in vitro and transforms them into indefinitely proliferating lymphoblastoid cell lines (LCLs). Because LCLs express a similar viral gene profile, it is considered as a model to study PTLDs and immune responses [12, 13].

EBV infects more than $90 \%$ of humans and produce latent infection for the life of host. However, persistent infection of EBV is basically benign in healthy carriers because EBV reactivation and subsequent proliferation of EBV-infected lymphocytes are suppressed by immunosurveillance. In contrast, in immunodeficient individuals, EBV can transform B cell into a proliferative state and eventually cause PTLDs $[14,15]$. It is considered that proliferation of the EBVinfected lymphocytes contained in the human cancer tissues occurs after xenotransplantation because of the insufficient immunosurveillance in the immunodeficient mice [6-10].

Lymphomagenesis after the xenotransplantation of human cancer tissues results in the loss of patient samples and waste of immunodeficient mice. However, it is still difficult to predict lymphomagenesis before xenotransplantation. We studied the pathological and molecular characteristics of surgically resected human CRC tissues, as well as both CRC PDXs and EBV-associated human lymphoma formed by their xenotransplantation. Our results suggest that the amount and/or presence of EBV itself in the cancer tissues for xenotransplantation are one of the factors that are associated with lymphomagenesis in the human cancer PDXs.

\section{RESULTS}

\section{Lymphomagenesis by the xenotransplantation of human CRC tissues}

To establish human CRC PDXs, we engrafted 13 fresh surgical specimens of CRC patients subcutaneously into the NOD/SCID or NSG mice and 9 PDXs were established. The clinicopathological features of the patients and the PDXs are presented in Table 1.

Histological examination showed that 7 of $9(78 \%)$ PDX tumors recapitulated histopathological characteristics of the patient CRC tissues (Supplementary Figure S1). But 2 PDXs (KUC4 and KUC11) did not resemble the parent CRC tissues but exhibited the morphological characteristics of lymphoma (Figure 1). Hematoxylin and eosin (H.E.) staining showed that both lymphoma tissues were composed of large atypical lymphoid cells arranged in a diffuse fashion, against the background of scattered small non-dysplastic lymphocytes. On immunostaining and in situ hybridization, the neoplastic cells were diffusely positive for a B cell marker CD20 and EBER, but negative for a $\mathrm{T}$ cell marker CD3 (Figure 1B). These morphological features were consistent with diffuse large B-cell lymphoma (DLBCL). Histology review of the patient CRC tissues revealed occasional small lymphoid aggregates consisting of mixed CD3-positive $\mathrm{T}$ lymphocytes and CD20-positive B lymphocytes, but neither dysplastic lymphocytes nor EBER-positive cells were found (Figure 1A). Flow cytometric analyses of KUC4 and KUC11 DLBCL PDXs showed that tumor cells within the PDXs were predominantly positive for human CD45 and negative for human EpCAM (Figure 2 ), confirming that these tumors originated from human lymphocytes presented in the surgical specimens.

DLBCL caused by proliferation of EBV-infected lymphocytes in human immunocompromised hosts metastasizes to distant organs. And DLBCL PDXs tend to form large metastases and grow faster than CRC PDXs which basically do not metastasize when grown subcutaneously in immunocompromised mice $[9,16]$. As shown in Table 1, the days for growth tended to be shorter in DLBCL PDX than in CRC PDX. We examined the distant organs of the KUC4 and KUC11 and found metastatic lymphoma in the lungs and liver of KUC11 mouse (Figure $3 \mathrm{~A}$ ), but not in KUC4 mouse. Histologically, the metastatic tumors consisted of diffusely arranged large lymphoid cells positive for EBER, the appearance nearly identical to the subcutaneous tumor. The tumor cells widely invaded into the adjacent liver and pulmonary parenchyma (Figure 3B).

\section{Clonal expansion of EBV-infected lymphocytes}

Because latent infection of EBV is most commonly observed in humans, we evaluated the presence of EBV by ISH for EBER. We found that both KUC4 and KUC11 were strongly positive for EBER (Figure 1B). In contrast, EBER ISH was negative for the lymphocytes within the patient specimens from which the DLBCL PDXs derived, suggesting that the amount of EBV was extremely low and below the detection threshold for EBER ISH (Figure $1 \mathrm{~A})$. We then analyzed the expression of EBNAs and LMP1 in the patient specimens and the DLBCL PDXs. We 
Table 1: Characteristics of PDX tumors and their corresponding CRC patients

\begin{tabular}{|c|c|c|c|c|c|c|}
\hline & PDX histology & Pathology & Preoperative chemotherapy & TNM $^{\mathbf{a}}$ & Mouse strain & Days for growth $^{b}$ \\
\hline KUC1 & $\mathrm{CRC}^{\mathrm{c}}$ & mod. ${ }^{\mathrm{c}}$ & None & pT3 pN1 cM1 & NOD/SCID & 75 \\
\hline KUC2 & $\mathrm{CRC}$ & mod. & None & pT4a pN0 cM1 & NOD/SCID & 30 \\
\hline KUC3 & No tumor & mod. & None & pT4a pN2 cM0 & NOD/SCID & - \\
\hline KUC4 & $\mathrm{DLBCL}^{\mathrm{c}}$ & mod. & None $^{\mathrm{d}}$ & pT2 pN0 cM0 & NOD/SCID & 28 \\
\hline KUC5 & $\mathrm{CRC}$ & mod. & None & pT3 pN0 cM1 & NOD/SCID & 176 \\
\hline KUC6 & $\mathrm{CRC}$ & mod. & None & pT3 pN1 cM0 & NSG & 223 \\
\hline KUC7 & $\mathrm{CRC}$ & mod. & None & pT2 pN0 cM0 & NSG & 35 \\
\hline KUC8 & CRC & mod. & None & pT4a pN1 cM1 & NSG & 47 \\
\hline KUC9 & CRC & mod. & None & pT4b pN0 cM0 & NSG & 19 \\
\hline KUC10 & No tumor & mod. & None & pT3 pN0 cM0 & NSG & - \\
\hline KUC11 & DLBCL & well. ${ }^{c}$ & None & pT3 pN1 cM0 & NSG & 55 \\
\hline KUC12 & No tumor & well. & None & pT3 pN1 cM1 & NSG & - \\
\hline KUC13 & No tumor & mod. & Yes & pT3 pN2 cM1 & NSG & - \\
\hline
\end{tabular}

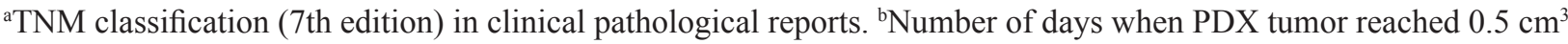
after xenotransplantation of patient specimen. ${ }^{c}$ Abbreviations: CRC, colorectal cancer; DLBCL, diffuse large B cell lymphoma; mod, moderately-differentiated adenocarcinoma; well, well-differentiated adenocarcinoma. ${ }^{\mathrm{d}}$ None, but under an immunosuppressive therapy after organ transplantation.

found that EBNA1, EBNA2, EBNA3C, and LMP1 were detectable in both DLBCL PDXs (Supplementary Figure S2), suggesting these lymphoma mimic DLBCL with type III latency. In the patient specimen of KUC11, EBNA 1, EBNA2, EBNA3C, and LMP1 were undetectable (Supplementary Figure S2). In contrast, the expression of EBNA 1, EBNA2, EBNA3C and LMP1 was detectable in the patient specimen of KUC4 (Supplementary Figure S2). We speculate that the upregulation of LMP1, a major transforming protein of $\mathrm{EBV}$, in the patient specimen of KUC4 affected the much faster growth of KUC4 DLBCL PDX than that of KUC11(Table 1, 28 days vs 55 days).

Then, we performed the clonality analyses of the lymphocytes by immunoglobulin heavy chain $(\operatorname{IgH})$ gene rearrangement assay. The unique dominant rearrangements that correspond to clonal B-cell proliferation, were detectable in the DLBCL PDXs, but not in the CRC PDXs (Figure 4), consistent with the notion that DLBCL is induced by the clonal expansion of the human $\mathrm{B}$ lymphocytes.

\section{PCR-based detection of EBV BamHI W region in patient cancer specimens correlates with lymphomagenesis}

EBV latent infection is highly prevalent among humans and relatively high lymphocyte infiltration are commonly observed in cancer tissues of human CRC specimens. Because the formation of DLBCL PDX by proliferation of EBV-infected lymphocytes results in the failure of the establishment of CRC PDXs, we examined the molecular factors that will be associated with the emergence of DLBCL PDXs.

The frequency of EBV-infected cells in peripheral blood of normal donors ranges approximately 1 in 10,000 to 1 in 100,000 memory B cells [17]. In the salivary gland, it is shown that there is less than 1 copy of EBV DNA per $1 \times 10^{5}$ cells [18]. Thus, we hypothesized that the difference of the amount of EBV in the surgical specimens affects the incidence of lymphomagenesis in the PDXs.

First, we analyzed the presence of EBV BamHI W region by PCR (EBV-BW PCR) in the patient specimens. Detection of BamHI W region, a major internal repeat in $\mathrm{EBV}$, is one of the most established methods to prove the presence of EBV genome [19]. We extracted $60 \mathrm{ng}$ genomic DNA from the surgical specimens of the CRC patients, except for that of KUC5 which we obtained was too small to be stored. EBV BamHI W region was amplified by EBVBW PCR in KUC4, but not in other 7 patient specimens analyzed (Figure 5A, upper panel). When the DNA samples were pre-amplified by 15 cycles and used as templates (p-EBV-BW PCR), we observed the amplification of EBV BamHI W region in 2 out of 7 patient specimens (KUC1 and KUC11, Figure 5A, middle panels). We confirmed that much higher amplification of EBV BamHI W region was observed using the DLBCL PDXs, KUC4 and KUC11, as templates (Figure 5B). When the EBV BamHI W region 


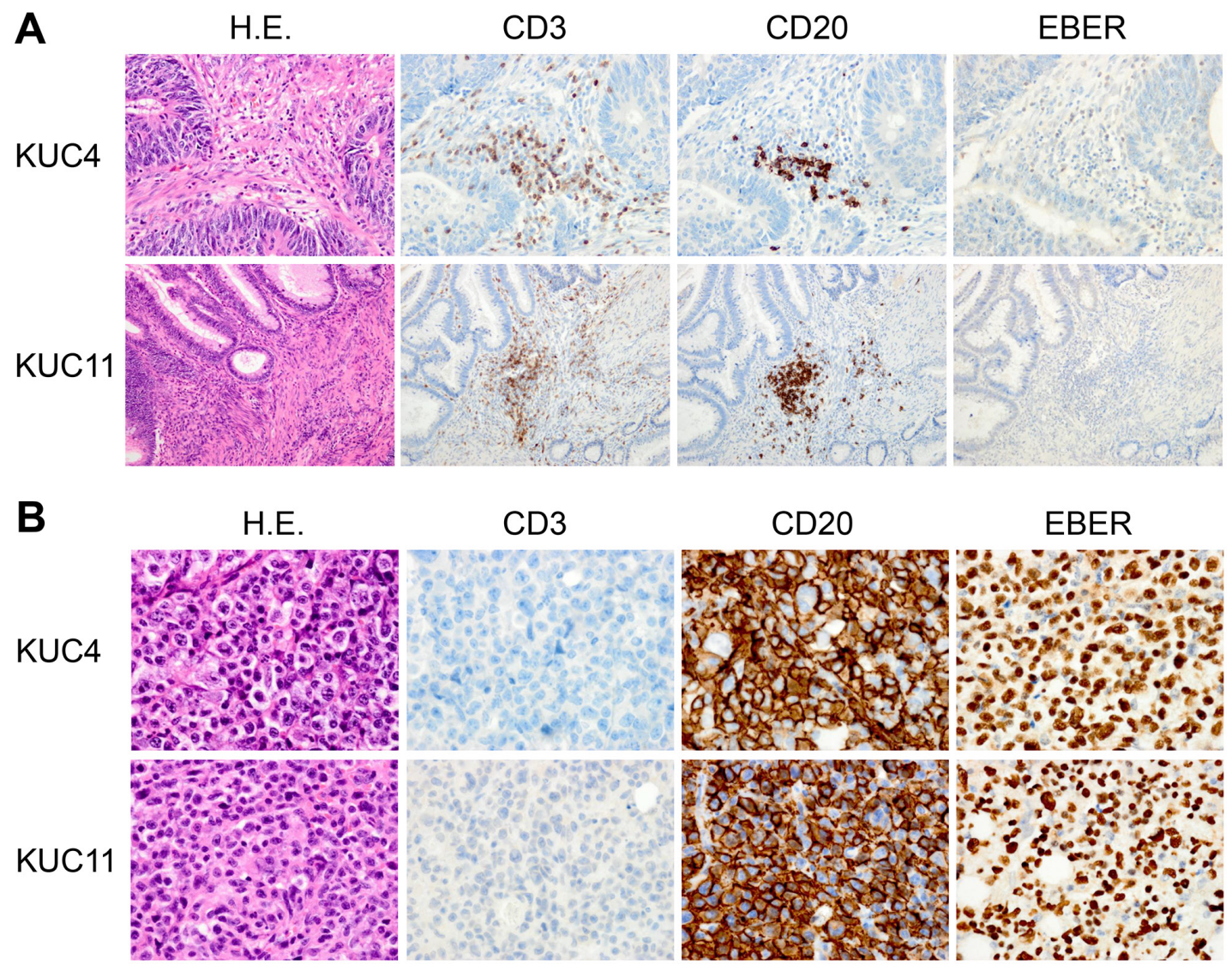

Figure 1: Establishment of the DLBCL PDXs from human CRC specimens. A. Representative sections of the patient CRC samples that gave rise to the DLBCL PDXs. H.E. (left panels), immunohistochemistry for CD3 and CD20 (middle panels), and EBER ISH (right panels) are shown (original magnification, $\times 200$ for KUC4, x100 for KUC11). Immunohistochemical analyses showed the infiltration of CD3-positive or CD20-positive lymphocytes in the tumor (brown, middle panels); but EBER was undetectable by ISH. B. Representative sections of the established DLBCL PDXs. H.E. (left panels), immunohistochemistry for CD3 and CD20 (middle panels), and EBER ISH (right panels) are shown (original magnification, $\times 400$ ). The cells in the PDXs were exclusively stained with an anti-CD20 antibody (brown). The cells were also strongly positive for EBER ISH (brown).

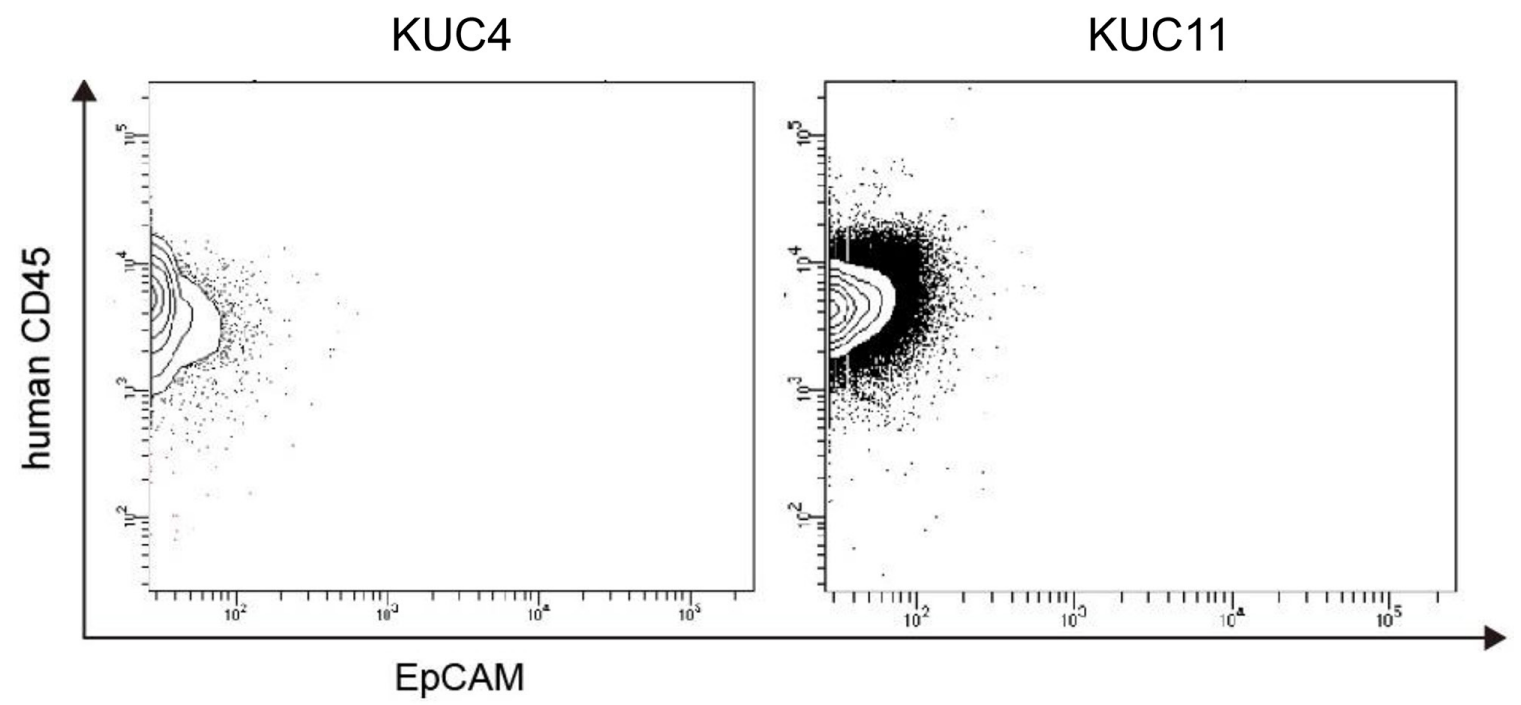

Figure 2: Flow cytometry analysis of the DLBCL PDXs. Representative flow cytometry profiles of the isolated cells from the DLBCL PDXs (KUC4 and KUC11). Most of the PDX tumor cells were within a human CD45 ${ }^{+}$EpCAM- population, which corresponds to human lymphocytes. 
was detectable by EBV-BW PCR or p-EBV-BW PCR, the transplantation of the samples resulted in lymphomagenesis in 2 out of 3 patient cancer specimens (Figure 5C). In contrast, when the EBV BamHI W region was undetectable by EBV-BW PCR or p-EBV-BW PCR, no patient cancer specimens resulted in lymphomagenesis ( 0 out of 7 patient cancer specimens). These result suggest that the PCR- detection of EBV in the patient $\mathrm{CRC}$ tissue is correlated with lymphomagenesis in the PDX.

Because the EBV-associated microRNAs are expressed in EBV-infected cells, we also analyzed the expression of BART 1-5p and BART 7 by RT-PCR. The expression levels of these miRNAs were extremely high in the DLBCL PDXs (Supplementary Figure S3), but were low

\section{A}

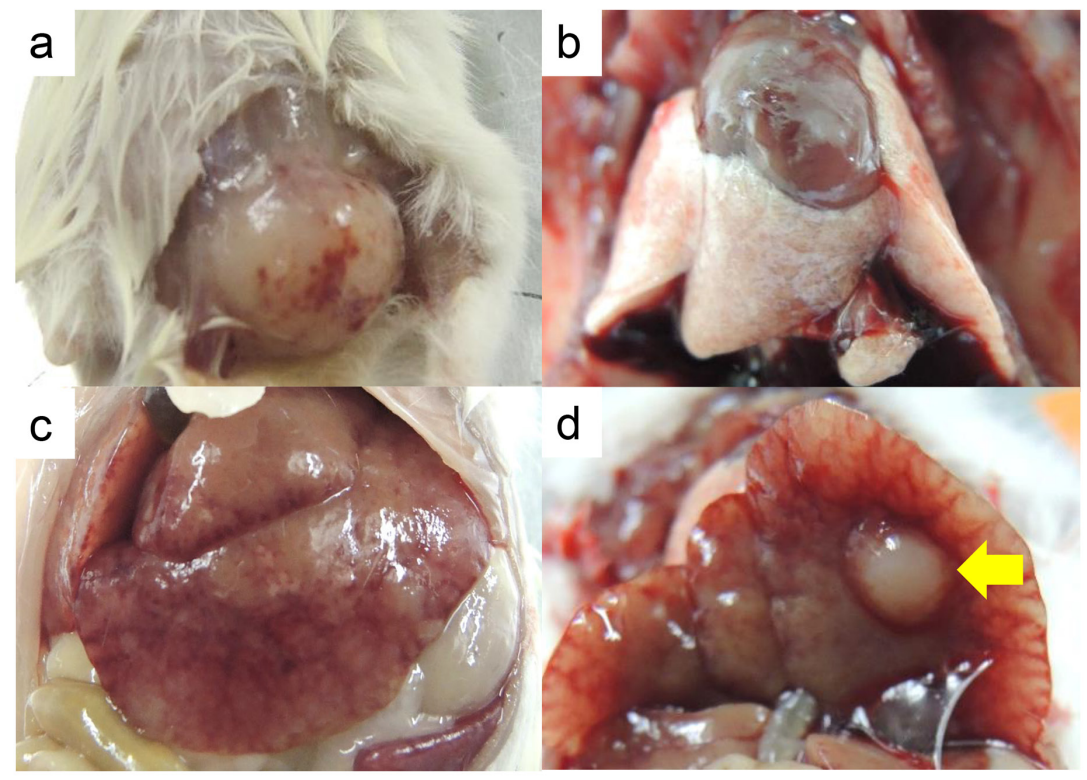

B

H.E.
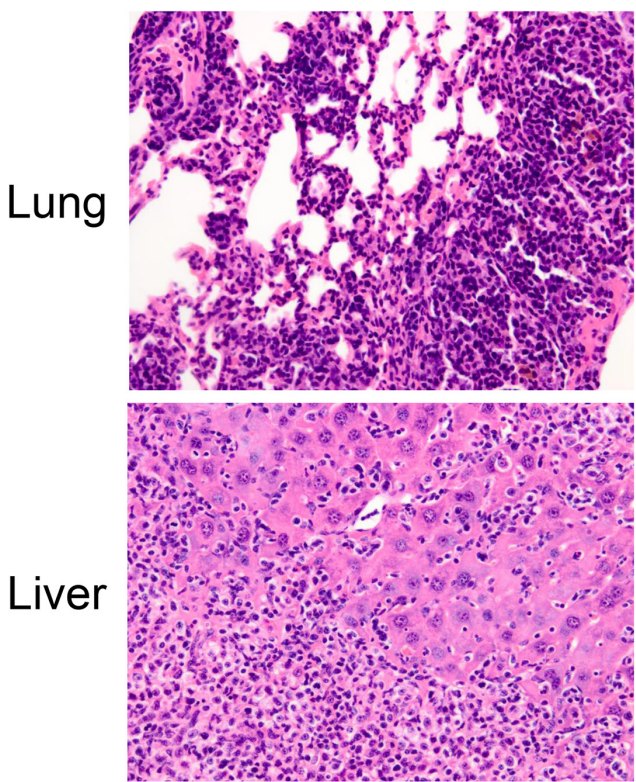

EBER
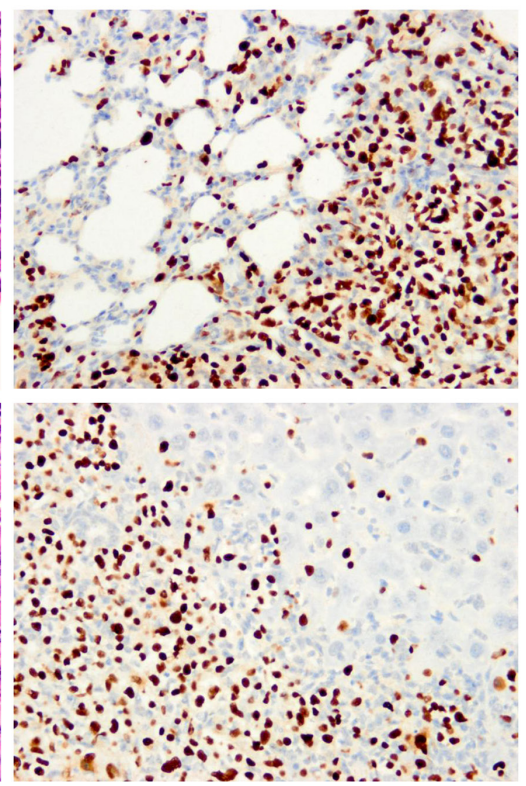

Figure 3: Formation of the DLBCL PDX and its distant metastases. A. Macroscopic views of the DLBCL PDX tumor and its metastatic organs (a. DLBCL PDX tumor, b. lung, c and d. liver). No tumor mass was grossly apparent in the lungs, while a large nodular metastasis was observed in the liver (yellow arrow). B. Diffuse infiltration of lymphoma cells in the lung and liver. H.E. (left panels) and EBER ISH (right panels) are shown (original magnification, $\times 100$ for the lung, $x 200$ for the liver). The infiltrated lymphocytes were strongly positive for EBER ISH (brown). 
or hardly detectable in the patient CRC specimen of KUC11 in which the amplification of the EBV BamHI W region was weak (Figure 5, Supplementary Figure S3). In contrast, these miRNAs were detectable in the patient specimen of KUC4 in which higher amplification of the EBV BamHI W region and the expression of EBNA genes were observed (Figure 5A, Supplementary Figures S2A and S3).

Taken together, these results suggest that the detection of EBV BamHI W region from the CRC surgical specimens with p-EBV-BW PCR will be an effective way to evaluate the risk of lymphomagenesis before xenotransplantation.

\section{DISCUSSION}

PDX model is an attractive model to analyze the character of cancer cells within the patient cancer tissues. However, establishment of PDX is occasionally hampered by the lymphomagenesis, mostly caused by proliferation

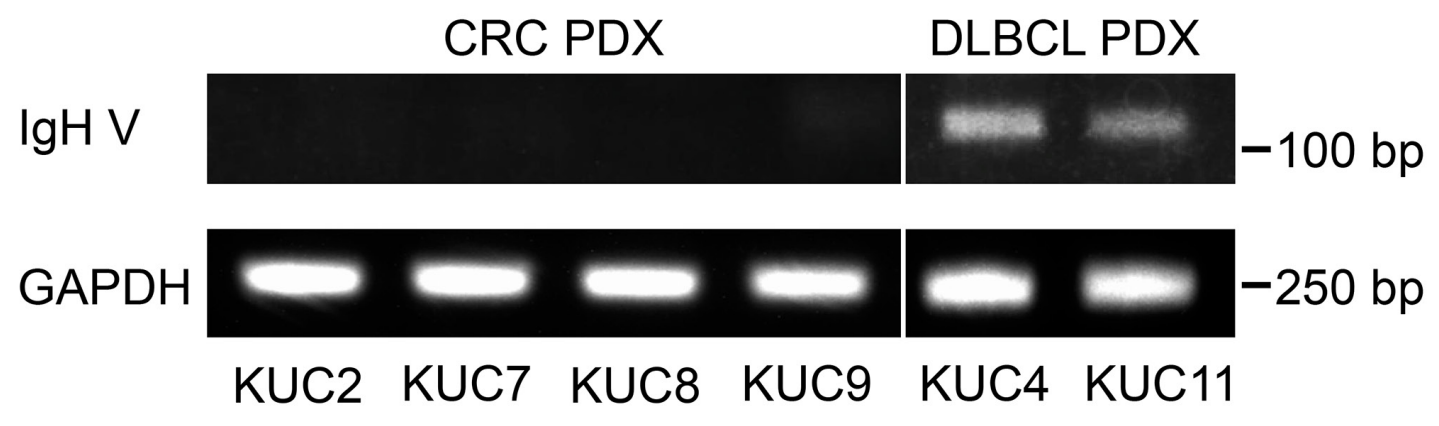

Figure 4: IgH gene rearrangements in the DLBCL PDXs. Presence of the dominant rearrangements of IgH gene in the PDXs was analyzed with the PCR amplification of the human $\operatorname{IgH}$ variable (IgH V) region. Unique dominant rearrangements that correspond to clonal B-cell proliferation were detectable in the DLBCL PDXs, but not in the CRC PDXs. GAPDH was amplified as an internal control.

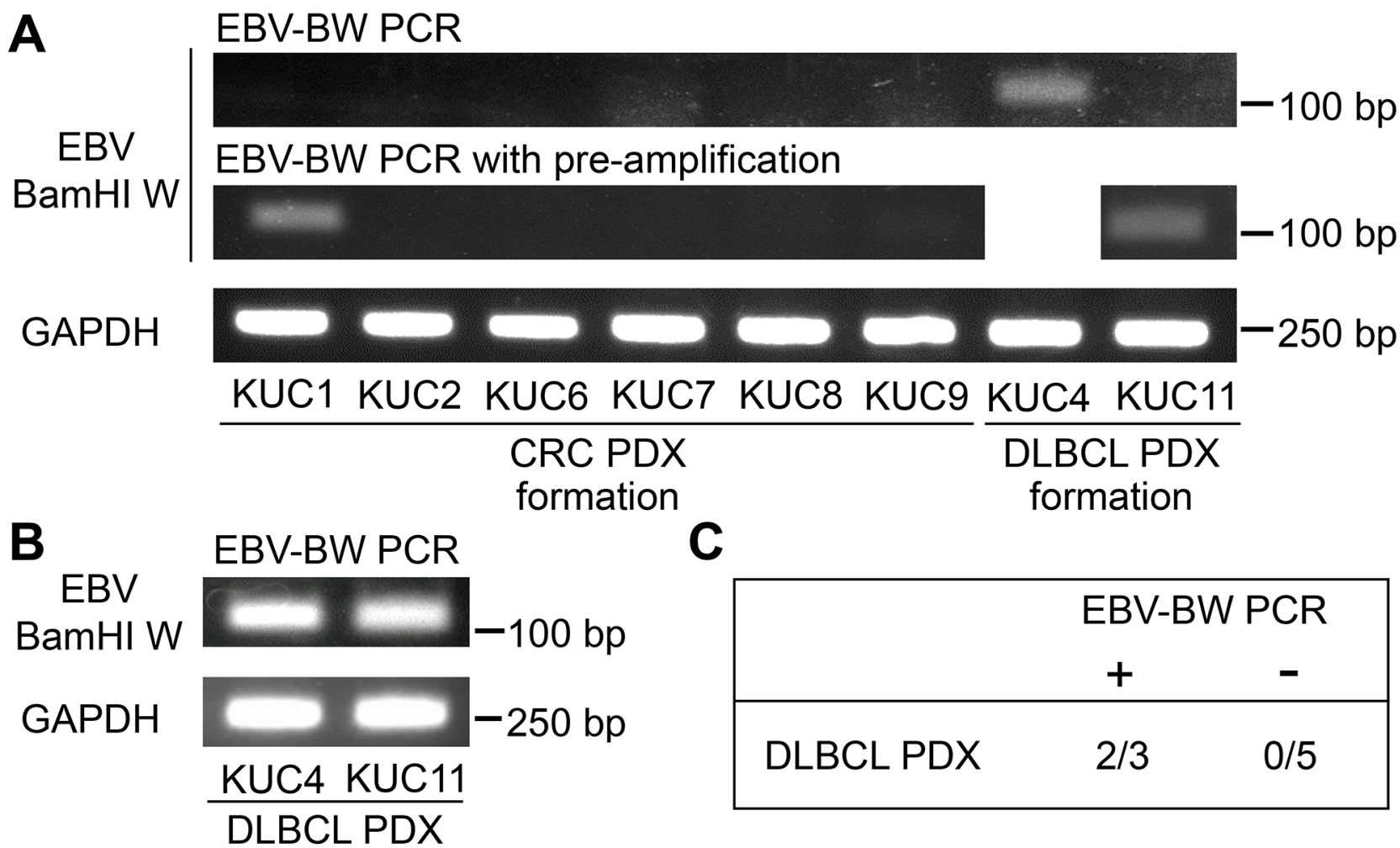

Figure 5: Amplification of EBV BamHI W region in the patient CRC specimens. A. RT-PCR analyses of the EBV BamHI W region in the patient $\mathrm{CRC}$ specimens which gave rise to the CRC or DLBCL PDXs. The amplification of BamHI W region was detected in 1 of 8 patient CRC specimens (KUC4) by EBV-BW PCR, and became detectable in 2 of 7 patient CRC specimens (KUC1 and KUC11) after the pre-amplification of templates (p-EBV-BW PCR). GAPDH was amplified as an internal control. B. RT-PCR analyses of the EBV BamHI W region in the DLBCL PDXs. BamHI W region was amplified by EBV-BW PCR. GAPDH was amplified as an internal control. C. Incidence of lymphomagenesis by the xenotransplantation of the patient CRC specimens positive or negative for EBV-BW PCR. Lymphomagenesis was observed in 2 of 3 EBV-BW PCR/p-EBV-BW PCR positive CRC specimens. 
of EBV-infected lymphocytes. Lymphomagenesis in the PDX is reported in other types of cancers, such as lung, liver, gastric, bladder, breast, prostate, and colorectal cancers [6-10]. Most of them exhibit histological character of EBV-associated DLBCL. In this study, we analyzed 13 human CRC tissues that resulted in the formation of 2 lymphomas and 7 histologically confirmed CRC PDXs. It has been reported that the tumor take rate of human primary CRC tissue in immunodecifient mice ranges between $63.5-84.4 \%$ which is relatively higher than other types of cancers, such as breast and prostate cancers $[1,20]$. Thus, the rate of establishment of CRC PDX (53.8\% (7/13)) and any PDXs including CRC and DLBCL PDXs $(69.2 \%(9 / 13))$ in this study is close to or within the range of the previous reports. In this study, 2 out of 9 PDXs showed morphological characteristics of lymphoma $(22.2 \%)$. Although the number of the reports on the lymphomagenesis in PDXs are still limited, other two reports present that the incidence of lymphomagenesis in the PDX transplanted with human CRC tissues was 3.3\% (1/30) and 28.6\% (2/7) [8, 9], suggesting that it is important to consider a possibilities of the lymphomagenesis when establishing PDX using the patient CRC tissues.

Several factors are reported to be associated with lymphomagenesis in the PDXs: low tumorigenicity of cancer cells in the patient primary tissues, preoperative chemotherapy, and inflammation in the tissues. In contrast, the factors, such as patient stage and vascular invasion, are not associated with the rate of lymphomagenesis. For example, the frequency of lymphomagenesis is up to $80 \%$ in the prostate cancer PDX which will be attributed to the low tumorigenicity of the androgen-sensitive prostate cancer [10]. Zhang et al. suggested that cancer type, preoperative chemotherapy and inflammation in patient primary cancer were associated with high risk of lymphomagenesis in PDX [8]. They suggested that gastric cancer specimens are more likely form DLBCL PDX than CRC ones, because of chronic mucosal inflammation caused by Helicobacter pylori. In this study which focused on $\mathrm{CRC}$, We found that KUC1 did not result in the formation of DLBCL lymphoma, even though the specimen was positive for $\mathrm{p}-\mathrm{EBV}-\mathrm{BW}$ PCR. We speculate that KUC1 specimen obtained from the patient of the metastatic CRC was more tumroigenic than other nonmetastatic cancer specimens, such as KUC4 and KUC11 (Table 1) and that lymphomagenesis was competitively suppressed by the engraftment and proliferation of CRC cells in the patient specimen.

The mechanism of lymphomagenesis in immunodeficient mice transplanted with human cancer is similar to that occur in severe immunodeficient patients, such as acquired immune deficiency syndrome and posttransplanted patients. Lymphoproliferative disorders in human immunodeficient patients are caused by the pathogens, such as EBV, HSV8, and HTLV-1. Among them, EBV is the dominant cause of lymphomagenesis in the PDXs reported to date [6-10]. EBV-associated lymphoma can be classified into the three latency types based on the expression of the latent EBV genes [21]. Briefly, type I, type II and type III latency are characterized by the expression of EBNA1, EBNA1 and LMP1, and EBNA 1, 2, 3 and LMP1, respectively. The expression profile of EBNA 1-3 and LMP1 confirmed that both of our DLBCL PDXs (KUC4 and KUC11) were latency type III lymphoma, which is observed in the patients under severe immunodeficient state. Consistent with our observation, the histology of other reported lymphomas is DLBCL. These results strongly suggest that the lymphoma PDXs are formed by proliferation of EBV-infected lymphocytes existed in the transplanted human cancer tissues. In this study, we analyzed the expression of BART miRNAs because BART miRNAs are reported as major contributors to the transformed growth properties of the EBV-infected cells [22]. The BART miRNAs can be detected by PCR in all forms of EBV latency, and their highest expression is detected in latency type I and II [23, 24]. We analyzed the expression level of the BART miRNA, such as BART 1-5p and BART7, in DLBCL PDX and corresponding patient CRC tissue. Consistent with their roles in transformation of lymphocytes, the expression levels of these miRNA were extremely high in the DLBCL PDXs (Supplementary Figure S3).

Although the expression of EBNAs and BART miRNAs were observed in the patient specimen of KUC4, this patient who received immunosuppressive therapies presented no clinical and pathological signs of lymphoma (Figure 1A, Table 1 Supplementary Figures S2 and $\mathrm{S} 3$ ). It is possible that proliferation of the EBV-infected lymphocytes was not induced in this patient at least partly because the expression of LMP1, an EBV genes required for transformation [25], was weak and was $10^{-3}$ times as much as that of the KUC4 DLBCL PDX (Supplementary Figure S2). Considering that the transplantation of KUC4 CRC specimen resulted in DLBCLPDX, it is possible that upregulation of the EBV genes in patient primary tissue is one of the factors that affect the lymphomagenesis in PDXs. On the other hand, because EBNA and LMP1 mRNAs and miRNAs were undetectable or very weakly expressed in all other patient specimens (Supplementary Figures S2 and S3), we could not correlate upregulation of the EBV genes and miRNAs in the patient specimens with the lymphomagenesis. Taken together, our results suggest that upregulation of EBV genes and miRNAs in patient tissue may not be associated with lymphomagenesis unless the patient is under severe immunosuppressive state.

In healthy EBV carriers, latent infection of EBV is basically limited to memory B cells that are in a resting state and no viral proteins are produced from the genome [26-28]. Because memory B cells exist in lymph nodes and rarely exist in blood and tissues, amount of EBV virus 
particles in the patient tissues may differ depending on the patient samples. In this study, we first applied EBV-BW PCR, to detect the presence of EBV in the patient cancer tissues for xenotransplantation. The presence of EBV is hardly detectable in the patient cancer tissues, except for KUC4, which was derived from the patient who received immunosuppressive therapies. To enhance the sensitivity of detection, we then pre-amplified the DNA templates and applied EBV-BW PCR. The p-EBV-BW PCR significantly enhanced the sensitivity of EBV detection and the additional 2 patient samples were positive for EBV.

Because $10 \mathrm{ng}$ of DNA is detectable in our standard agarose gel electrophoresis and the p-EBV-BW PCR method amplifies DNA $2.3 \times 10^{11}$ times in theory, the presence of $4.3 \times 10^{-20} \mathrm{~g}$ of DNA (about 40 base pairs) in the template is ideally detectable. Considering that the length of the BamHI W region is 125 base pairs and EBV genome contains multiple copies of BamHI W, we speculate that the presence of even $1 \mathrm{EBV}$ particle in the patient cancer sample can be theoretically detectable by the p-EBV-BW PCR method. Lymphoma were formed by the patient CRC samples positive for EBV-BW-PCR and/ or p-EBV-BW-PCR, and no lymphoma was formed by the patient samples negative for $\mathrm{p}-\mathrm{EBV}-\mathrm{BW}-\mathrm{PCR}$. Although further studies are required, these results suggest that the relative amount of EBV and/or the presence of the EBV virus particle itself in the patient sample are an important factor(s) for lymphomagenesis after xenotransplantation.

In summary, the amount of EBV in the patient cancer tissues significantly differs depending on the patients and the relative amount of EBV in the patient sample is one of the important factors that affect lymphomagenesis after xenotransplantation. To our knowledge, p-EBV-BW PCR is the first method to evaluate the risk of lymphomagenesis before xenotransplantation to prevent the loss of patient samples. Considering that no lymphoma was formed after transplantation of the patient samples negative for p-EBV-BW PCR and all lymphoma was formed from the samples positive for EBV-BW PCR/p-EBV-BW PCR, we propose that additional strategies, such as the depletion of lymphocytes from the patient samples, will be better to be performed before xenotransplantation when the patient cancer samples are positive for p-EBV-BW PCR.

\section{MATERIALS AND METHODS}

\section{Patient samples}

Primary human CRC tissues were obtained from the patients who admitted Kobe University Hospital. The written informed consent was obtained from each patient. The cancer type, histological grade and clinical stage (TNM classification, 7th edition) were obtained from clinical and histopathological reports. The investigation was preapproved by the Institutional Review Boards at Kobe University (permission number: 1299).

\section{Xenografts}

All animal experiments were performed under the approval of the Kobe University Animal Care and Use Committee (permission number: 150802). All surgical procedures were performed under isoflurane anesthesia with care to minimize suffering of mice. The tissues obtained from CRC patients were minced and suspended in Matrigel (BD Biosciences), and then subcutaneously transplanted on the flank of female NOD/SCID or NSG mice (Oriental Kobo, Japan) as previously described [29, 30]. Nine PDX lines were established with the method. When the tumor reached approximately $1-2 \mathrm{~cm}$ in size, the tumors were harvested for analyses.

\section{Histological examination}

A portion of the patient CRC tissues were formalin fixed for the pathological evaluation. The PDX tissues were fixed in $4 \%$ paraformaldehyde/phosphate buffered saline (PBS) for 8 hours at $4^{\circ} \mathrm{C}$. The paraffinembedded specimens were cut and stained with H.E.. Immunostaining of CD3 or CD20 was performed by an autostainer (HX System Benchmark, Ventana Medical Systems, Tucson, AZ). Primary antibodies used were a mouse monoclonal antibody for human CD3 (1:50, Dako Cytomation, Glostrup, Denmark) and a mouse monoclonal antibody for human CD20 (1:50, Dako Cytomation). The in situ hybridization of EBER was also conducted by the autostainer according to the manufacturer's instructions (HX System Benchmark, Ventana Medical Systems). A specific probe for EBER was obtained from Ventana Medical Systems.

\section{Flow cytometry analysis}

PDX tissues were dissociated as previously described [31]. Briefly, the tumor tissues were dissociated with collagenase III (Worthington Biochemical Corporation) and were blocked with normal mouse IgG (1:100, Wako). The cells were stained with FITCconjugated anti-EpCAM (1:10, Biolegend), and PE-Cy7conjugated anti-human CD45 (1:100, BD) antibodies. Mouse cells and dead cells were depleted by using anti-mouse $\mathrm{H}-2 \mathrm{Kd}$ (1:40, eBioscience) and biotinconjugated anti-CD45 (1:40, BD) antibodies and PECy-5 streptavidin, and 4',6-diamidino-2-phenylindole, respectively. The profile was analyzed using a FACS Aria III cell sorter (BD Biosciences).

\section{IgH gene rearrangement assay}

DNA was isolated from a portion of the patient primary CRC and the PDX tissues using DNeasy Blood \& Tissue Kit (QIAGEN) according to the manufacturer's instructions. Clonality of the lymphocytes within the PDXs was analyzed by PCR-based $\operatorname{IgH}$ gene rearrangement 
assay using the primers as previously described [7]. PCR that amplifies the $\mathrm{IgH}$ variable region was performed with KOD DNA polymerase Mix (TOYOBO). Amplification fragment were visualized with ethidium bromide agarose gel electrophoresis. Clonality was determined by the presence of single and unique sized band. PCR of glyceraldehyde-3-phosphate dehydrogenase (GAPDH) was performed as a control using the following GAPDH primers: forward 5'-AGAAGGCTGGGGCTCATTTG-3', and reverse 5'-AGGGGCCATCCACAGTCTTC-3'.

\section{Pre-amplification and PCR-based assay of the EBV-BamHI W region}

EBV BamHI W region was amplified by PCR using the $60 \mathrm{ng}$ genomic DNA which was extracted from the patient primary CRC or the PDX tissue as a template. Primers used were as follows: forward 5'-CGGTCGCCCAGTCCTACCAG-3', and reverse 5'-CCTGGAGAGGTCAGGTTACT-3'. Pre-amplification was performed using the same primer sets when necessary. Pre-amplified sample was diluted 8 times before the PCR amplification of the EBV BamHI W region. The protocol for the pre-amplification was follows: at $98^{\circ} \mathrm{C}$ for 2 minutes, and then followed by 15 cycles of $98^{\circ} \mathrm{C}$ for 2 minutes, $56^{\circ} \mathrm{C}$ for 10 seconds and $68^{\circ} \mathrm{C}$ for 30 seconds. PCR was performed with KOD DNA polymerase Mix as follows: at $98^{\circ} \mathrm{C}$ for 2 minutes, and then followed by 30 cycles of $98^{\circ} \mathrm{C}$ for 10 seconds, $53^{\circ} \mathrm{C}$ for 10 seconds and $68^{\circ} \mathrm{C}$ for 20 seconds. Amplified fragments were visualized with ethidium bromide agarose gel electrophoresis.

\section{Semi-quantitative reverse transcription PCR}

RNA was extracted from the patient CRC and PDX tissues using Trizol Reagent (Invitrogen) according to the manufacturer's instructions. After the reverse transcription (RT) using MultiScrib Reverse Transcriptase (Applied Biosystems), PCR were performed using primer sets as follows: EBNA1 forward 5'-GGATGCGATTAAGGACCTTGTT-3', and reverse 5'-CGTCAAAGCTGCACACAGTC-3'; EBNA2 forward 5'-CTACTCACGGTACTACAAAGGC-3', and reverse 5'-CCGTGGTTCTGGACTATCTGGA-3'; EBNA3C forward 5'-CAAGGTGCATTTACCCCACTG-3', and reverse 5'-GGGCAGGTCCGTGAGAACT-3'; LMP1 forward 5' - CCTTGGTCTACTCCTACTGATG-3', and reverse 5' - TTACCAAGTAAGCAGCCAAAGATG-3'; GAPDH forward 5'-AGAAGGCTGGGGCTCATTTG-3', and reverse 5'-AGGGGCCATCCACAGTCTTC-3'. PCR was performed as follows: at $98^{\circ} \mathrm{C}$ for 2 minutes, and then followed by 40 cycles of $98^{\circ} \mathrm{C}$ for 10 seconds, $52^{\circ} \mathrm{C}$ for 10 seconds and $68^{\circ} \mathrm{C}$ for 20 seconds. Data were normalized to the amount of GAPDH expression.

Expression of EBV miRNAs, BART 1-5p and BART 7 were analyzed by semi-quantitative RT- PCR as previously described [32]. The primers were as follows: BART 1-5p RT 5'-CTCAACTGGTGTCGTGGAGTCG GCATTCAGTTGAGACAGCACG-3', forward 5'-AC ACTCCAGCTGGGTCTTAGTGGAAGTGAC-3', and probe 5'-TTCAGTTGAGACAGCACG-3'; BART 7 RT 5'-CTCAACTGGTGTCGTGGAGTCGGCAATT CAGTTGAGCCCTGGAC-3', forward 5'-ACACTC CAGCTGGGCATCATAGTCCAGTGT-3', and probe 5'-TTCAGTTGAG CCCTGGAC-3'; universal reverse 5'-CAACTGGTGTCGTGGAGTCGGCAA-3'. Realtime PCR was performed in the Thermal Cycler Dice (TAKARA), as follows: at $50^{\circ} \mathrm{C}$ for 2 minutes and $98^{\circ} \mathrm{C}$ for 10 minutes, and then followed by 40 cycles of $95^{\circ} \mathrm{C}$ for 15 seconds and $60^{\circ} \mathrm{C}$ for 1 minute. Data were normalized by the amount of small nuclear RNA expression, SNORD 48.

\section{ACKNOWLEDGMENTS}

The authors gratefully acknowledge the patients who accepted to contribute to this research and their family. We also thank the division of Gastrointestinal Surgery of Kobe University Graduate School of Medicine for contribution in the patient tumor collection, and Hiroki Kondo and Yasuteru Tanaka, medical students of Kobe University, for technical assistance.

\section{CONFLICTS OF INTEREST}

The authors declare that there are no conflicts of interest.

\section{GRANT SUPPORT}

This study was supported by Grants-in-Aid for Scientific Research from the Japan Society of the Promotion of Science and by the grants from Japan Foundation for Applied Enzymology and the Itoh-Chubei Foundation.

\section{REFERENCES}

1. Julien S, Merino-Trigo A, Lacroix L, Pocard M, Goéré D, Mariani P, Landron S, Bigot L, Nemati F, Dartigues P, Weiswald LB, Lantuas D Morgand L et al. Characterization of a large panel of patient-derived tumor xenografts representing the clinical heterogeneity of human colorectal cancer. Clin Cancer Res. 2012; 18:5314-5328.

2. Nobutani K, Shimono Y, Mizutani K, Ueda Y, Suzuki T, Kitayama M, Minami A, Momose K, Miyawaki K, Akashi $\mathrm{K}$, Azuma T and Takai Y. Downregulation of CXCR4 in Metastasized Breast Cancer Cells and Implication in Their Dormancy. PLoS One. 2015; 10: e0130032.

3. Williams SA, Anderson WC, Santaguida MT and Dylla SJ. Patient-derived xenografts, the cancer stem cell paradigm, 
and cancer pathobiology in the 21 st century. Lab Invest. 2013; 93:970-982.

4. Whittle JR, Lewis MT, Lindeman GJ and Visvader JE. Patient-derived xenograft models of breast cancer and their predictive power. Breast Cancer Res. 2015; 17:17.

5. Gupta SK, Kizilbash SH, Carlson BL, Mladek AC, BoakyeAgyeman F, Bakken KK, Pokorny JL, Schroeder MA, Decker PA, Cen L, Eckel-Passow JE, Sarkar G Ballman $\mathrm{KV}$, et al. Delineation of MGMT Hypermethylation as a Biomarker for Veliparib-Mediated TemozolomideSensitizing Therapy of Glioblastoma. J Natl Cancer Inst. 2015; 108: djv369.

6. John T, Yanagawa N, Kohler D, Craddock KJ, BandarchiChamkhaleh B, Pintilie M, Sykes J, To C, Li M, Panchal D, Chen W, Shepherd FA and Tsao MS. Characterization of lymphomas developing in immunodeficient mice implanted with primary human non-small cell lung cancer. J Thorac Oncol. 2012; 7: 1101-1108.

7. Chen K, Ahmed S, Adeyi O, Dick JE and Ghanekar A. Human solid tumor xenografts in immunodeficient mice are vulnerable to lymphomagenesis associated with EpsteinBarr virus. PLoS One. 2012; 7: e39294.

8. Zhang L, Liu Y, Wang X, Tang Z, Li S, Hu Y, Zong X, Wu X, Bu Z, Wu A, Li Z, Li Z, Huang X et al. The extent of inflammatory infiltration in primary cancer tissues is associated with lymphomagenesis in immunodeficient mice. Sci Rep. 2015; 5:9447.

9. Bondarenko G, Ugolkov A, Rohan S, Kulesza P, Dubrovskyi O, Gursel D, Mathews J, O’Halloran TV, Wei JJ and Mazar AP. Patient-derived tumor xenografts are susceptible to formation of human lymphocytic tumors. Neoplasia. 2015; 17:735-741.

10. Wetterauer C, Vlajnic T, Schüler J, Gsponer JR, Thalmann GN, Cecchini M, Schneider J, Zellweger T, Pueschel H, Bachmann A, Ruiz C, Dirnhofer S, Bubendorf L, et al. Early development of human lymphomas in a prostate cancer xenograft program using triple knock-out immunocompromised mice. Prostate. 2015; 75:585-592.

11. Rickinson $\mathrm{AB}$ and Kieff E Epstein-Barr virus. In: Fields BN, Kniple DM, Howley PM, editors. Fields Virology, 4th edn. Lippincott Williams and Wilkins: Philadelphia, PA. 2001; pp 2575-2627.

12. Young LS and Rickinson AB. Epstein-Barr virus: 40 years on. Nat Rev Cancer. 2004; 4:757-768.

13. Hislop AD, Taylor GS, Sauce D and Rickinson AB. Cellular responses to viral infection in humans: lessons from Epstein-Barr virus. Annu Rev Immunol. 2007; 25:587-617.

14. Thorley-Lawson DA and Gross A. Persistence of the Epstein-Barr virus and the origins of associated lymphomas. N Engl J Med. 2004; 350: 1328-1337.

15. Epeldegui M, Vendrame E and Martínez-Maza O. HIVassociated immune dysfunction and viral infection: role in the pathogenesis of AIDS-related lymphoma. Immunol Res. $2010 ; 48: 72-83$
16. Hoffman RM. Patient-derived orthotopic xenografts: better mimic of metastasis than subcutaneous xenografts. Nat Rev Cancer. 2015; 15:451-452.

17. Laichalk LL, Hochberg D, Babcock GJ, Freeman RB, Thorley-Lawson DA. The dispersal of mucosal memory B cells: evidence from persistent EBV infection. Immunity. 2002; 16:745-754.

18. Saito I, Servenius B, Compton T and Fox RI. Detection of Epstein-Barr virus DNA by polymerase chain reaction in blood and tissue biopsies from patients with Sjogren's syndrome. J Exp Med. 1989; 169:2191-2198.

19. Baer R, Bankier AT, Biggin MD, Deininger PL, Farrell PJ, Gibson TJ, Hatfull G, Hudson GS, Satchwell SC, Séguin C, Tuffnell PS and Barrell BG. DNA sequence and expression of the B95-8 Epstein-Barr virus genome. Nature. 1984; 310:207-211.

20. Puig I, Chicote I, Tenbaum SP, Arqués O, Herance JR, Gispert JD, Jimenez J, Landolfi S, Caci K, Allende H, Mendizabal L, Moreno D, Charco R, et al. A personalized preclinical model to evaluate the metastatic potential of patient-derived colon cancer initiating cells. Clin Cancer Res. 2013; 19:6787-6801.

21. Niller HH, Wolf $\mathrm{H}$ and Minarovits J. Regulation and dysregulation of Epstein-Barr virus latency: implications for the development of autoimmune diseases. Autoimmunity. 2008; 41:298-328.

22. Marquitz AR, Mathur A, Shair KH and Raab-Traub N. Infection of Epstein-Barr virus in a gastric carcinoma cell line induces anchorage independence and global changes in gene expression. Proc Natl Acad Sci U S A. 2012; 109:9593-9598.

23. Cai X, Schäfer A, Lu S, Bilello JP, Desrosiers RC, Edwards $\mathrm{R}$, Raab-Traub $\mathrm{N}$ and Cullen BR. Epstein-Barr virus microRNAs are evolutionarily conserved and differentially expressed. PLoS Pathog. 2006; 2: e23.

24. Edwards RH, Marquitz AR and Raab-Traub N. Epstein-Barr virus BART microRNAs are produced from a large intron prior to splicing. J Virol. 2008; 82:9094-9106.

25. Young LS and Murray PG. Epstein-Barr virus and oncogenesis: from latent genes to tumours. Oncogene. 2003; 22:5108-5121.

26. Babcock GJ, Decker LL, Freeman RB and Thorley-Lawson DA. Epstein-barr virus-infected resting memory B cells, not proliferating lymphoblasts, accumulate in the peripheral blood of immunosuppressed patients. J Exp Med. 1999; 190:567-576.

27. Miyashita EM, Yang B, Babcock GJ and ThorleyLawson DA. Identification of the site of Epstein-Barr virus persistence in vivo as a resting B cell. J Virol. 1997; 71:4882-4891.

28. Hochberg D, Middeldorp JM, Catalina M, Sullivan JL, Luzuriaga K and Thorley-Lawson DA. Demonstration of the Burkitt's lymphoma Epstein-Barr virus phenotype in dividing latently infected memory cells in vivo. Proc Natl Acad Sci U S A. 2004; 101:239-244. 
29. DeRose YS, Wang G, Lin YC, Bernard PS, Buys SS, Ebbert MT, Factor R, Matsen C, Milash BA, Nelson E, Neumayer L, Randall RL, Stijleman IJ, et al. Tumor grafts derived from women with breast cancer authentically reflect tumor pathology, growth, metastasis and disease outcomes. Nat Med. 2011; 17:1514-1520.

30. Shimono Y, Zabala M, Cho RW, Lobo N, Dalerba P, Qian D, Diehn M, Liu H, Panula SP, Chiao E, Dirbas FM, Somlo G, Pera RA, et al. Downregulation of miRNA-200c links breast cancer stem cells with normal stem cells. Cell. 2009; 138:592-603.
31. Cho RW, Wang X, Diehn M, Shedden K, Chen GY, Sherlock G, Gurney A, Lewicki J and Clarke MF. Isolation and molecular characterization of cancer stem cells in MMTV-Wnt-1 murine breast tumors. Stem Cells. 2008; 26:364-371.

32. Cosmopoulos K, Pegtel M, Hawkins J, Moffett H, Novina C, Middeldorp $\mathrm{J}$ and Thorley-Lawson DA. Comprehensive profiling of Epstein-Barr virus microRNAs in nasopharyngeal carcinoma. J Virol. 2009; 83:2357-2367. 